\title{
Finanzkrise, soziale Ungleichheit und Infektionskrankheiten
}

\author{
Tim Eckmanns
}

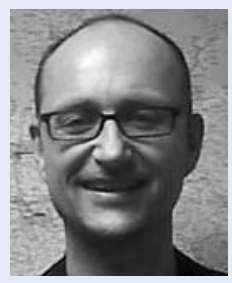

Tim Eckmanns
„....Politik ist nichts weiter als Medizin im Großen.“

Rudolf Virchow (1848)

Im Rahmen der weltweiten Finanzkrise (in den Jahren 2008 ff.) wurden harte ökonomische Entscheidungen gegenüber Ländern bzw. den dort Lebenden getroffen, die oft nicht dem medizinischen Grundsatz „primum non nocere" (zuerst einmal nicht schaden) folgten. In einer systematischen Übersichtsarbeit beschreiben Suhrcke et. al. die Folgen von ökonomischen Krisen für die Verbreitung übertragbarer Krankheiten [1]. Sie zeigen, dass den meisten Studien zufolge (30 von 37) die Zahl der Infektionskrankheiten im Rahmen der Krise angestiegen sind. Als Erklärung legten sie das SIRModel (susceptible, infectious, recovered) zugrunde. Dem zufolge sind die Menschen auf Grund von eingeschränkter Nahrung, einem angegriffenem Immunsystem und schlechter Immunisierung im Zeitraum der Krise empfänglicher für Infektionskrankheiten. Die Infektionsrate steigt direkt z.B. in Folge überfüllter Gefängnisse, der Zunahme von Hochrisikopopulationen wie z. B. Obdachlosen oder schlechterer Wohnbedingungen, aber auch indirekt in Folge des Zusammenbruchs der Infrastruktur (z. B. Kurzschlüsse zwischen Abwasser und Wasserversorgung) oder des Anstiegs von Vektoren. Gleichzeitig ist während der Krise die Therapie eingeschränkt, da die Anzahl der Ärzte sinkt und Medikamente nicht mehr erschwinglich sind.

Hinweise auf Zusammenhänge zwischen der krisenhaften Entwicklung und der Zunahme von Infektionskrankheiten lassen sich auch am Beispiel von Griechenland beobachten [2]. Griechenland hatte in der Pandemie A (H1N1) 2009/10 eine der höchsten Mortalitätsraten in Europa. In den Jahren 2010 und 2011 gab es erstmals einen West Nile-Virus-Ausbruch in Griechenland mit zahlreichen Todesfällen. Auch kam es von 2009 bis 2011 zu einem Malaria-Plasmodium-vivaxAusbruch. Ursache für diese Ausbrüche war offenbar das Zurückfahren der Public Health Maßnahmen im Bereich der Mückenbekämpfung. Zusätzlich kam es im Jahr 2011 zu einem 15-fachen Anstieg von neu diagnostizierten HIV Fällen unter i.v.-Drogenabhängigen.
Zum Weg der Austeritätspolitik wie in Griechenland existieren Alternativen. So haben Studien gezeigt, dass gerade auch im Bereich Gesundheit Investitionen während der Krise sich mittel- und langfristig positiv auswirken können $[3,4,5]$. Island hat sich nicht die Strukturpolitik aufzwingen lassen und steht jetzt gesundheitspolitisch besser da als Griechenland, dem harte Maßnahmen aufoktroyiert wurden und das diese auch umgesetzt hat.

Aber auch in der Abwesenheit von ökonomischen Krisen sind sozial benachteiligte Gruppen vermehrt von Infektionskrankheiten betroffen. In einem Review von Semenza et al. konnte dies für alle EU Mitgliedstaaten gezeigt werden [6]. So ist z.B. die Wahrscheinlichkeit in Berlin bei Kindern an Rotaviren zu erkranken, in einer Umgebung mit einer höheren Anzahl an Arbeitslosen, größer als mit weniger Arbeitslosen [7]. Allerdings ist es oft schwierig, patientenbezogene Daten zum sozialen Status zu erheben und daher werden meist nur ökologische Studien durchgeführt, so dass ein direkter Rückschluss vom Sozialstatus auf Infektionskrankheit offen bleibt.

Zum Zusammenhang von Sozialstatus und nosokomialen Infektionen existieren nur sehr wenige Studien. In einer ökologischen Studie zu postoperativen Wundinfektionen mit MRSA konnte gezeigt werden, dass Patienten aus den am stärksten sozial benachteiligten Regionen des Vereinigten Königreichs eine siebenfach höhere Infektionsrate haben als Patienten aus den am wenigsten benachteiligten Regionen [8]. In Deutschland existieren dem gegenüber noch keine Studien zum Zusammenhang zwischen Sozialstatus und nosokomialen Infektionen.

Im Oktober fand an der europäischen Seuchenbekämpfungsbehörde - European Centre for Disease Prevention and Control (ECDC) - ein Workshop zum Thema „Health inequalities, financial crisis, and infectious disease control in the EU' (Gesundheitliche Ungleichheit, Finanzkrise und Prävention von Infektionskrankheiten) statt. In den Diskussionen wurden die Auswirkungen von sozialer Ungleichheit wie auch von ökonomischen Krisen auf Infektionskrankheiten thematisiert. 
Folgende Vorschläge für die Mitgliedsländer wurden in den Raum gestellt:

1. In jedem Studiendesign soll die Frage nach sozialen Unterschieden genauso adressiert werden, wie dies jetzt für Geschlechterunterschiede der Fall ist.

2. Jede Public Health Maßnahme, die neu eingeführt wird, muss daraufhin untersucht werden, ob sie alle sozialen Milieus bzw. Schichten erreicht - oder ob sie zur Vergrößerung der Gesundheitsunterschiede zwischen Bevölkerungsgruppen mit einem hohem und einem niedrigem sozialen Status führt, da v.a. die Mittel- und Oberschicht von der Maßnahme profitiert.

3. Es gilt, alle Infektionskrankheiten, insbesondere auch die von Flüchtlingen, zu behandeln.

Die Schlussfolgerung erscheint eindeutig, Fragen sozialer Ungleichheit dürfen von einer aktuellen Public Health Politik nicht mehr vernachlässigt werden. Ein Umdenken ist vonnöten.

\section{Literatur}

1 Suhrcke M, Stuckler D, Suk JE et al. The impact of economic crises on communicable disease transmission and control: a systematic review of the evidence. PloS one 2011; 6: e20724

2 Bonovas S, Nikolopoulos G. High-burden epidemics in Greece in the era of economic crisis. Early signs of a public health tragedy. J Prev Med Hyg 2012; 53: 169 - 171

3 Stuckler D, Basu S, McKee M. How government spending cuts put lives at risk. Nature 2010; 465: 289

4 McKee M, Suhrcke M, Nolte E et al. Health systems, health, and wealth: a European perspective. Lancet 2009; 373: 349 351

5 Reeves A, Basu S, McKee M et al. Does investment in the health sector promote or inhibit economic growth? Global Health 2013; 9: 43

6 Semenza JC, Giesecke J. Intervening to reduce inequalities in infections in Europe. Am J Public Health 2008; 98: 787 - 792

7 Wilking $\mathrm{H}$, Höhle $\mathrm{M}$, Velasco E et al. Ecological analysis of social risk factors for Rotavirus infections in Berlin, Germany, 2007-2009. Int J Health Geogr 2012; 11: 37

8 Bagger JP, Zindrou D, Taylor KM. Postoperative infection with meticillin-resistant Staphylococcus aureus and socioeconomic background. Lancet 2004; 363: 706 - 708 